\title{
Somatotopic or spatiotopic? Frame of reference for localizing thermal sensations under thermo-tactile interactions
}

\author{
Hsin-Ni Ho \\ NTT Communication Science Laboratories, Atsugi, Japan \\ JUNJI WaTANABE \\ NTT Communication Science Laboratories, Atsugi, Japan \\ and Japan Society for the Promotion of Science, Atsugi, Japan \\ Hideyuki ANDo \\ NTT Communication Science Laboratories, Atsugi, Japan \\ AND \\ MaKio Kashino \\ NTT Communication Science Laboratories, Atsugi, Japan \\ and Tokyo Institute of Technology, Yokohama, Japan
}

\begin{abstract}
The thermal sense is diffuse and incapable of providing precise spatial information. From a phenomenon known as thermal referral, we know that touch influences the localization of cold or warmth, leading to our perceiving illusory thermal sensations at a thermally neutral site. This study investigated the frame of reference for localizing thermal sensations under thermal referral in order to shed light on how thermal and tactile modalities coordinate to process localization information. One thermally neutral tactile stimulator and two cold (warm) stimulators were presented to different sets of three fingers of both hands. The location of the neutral tactile stimulator varied, and the strength of the referral was estimated from participants' performance in localizing the neutral tactile stimulator. By manipulating the somatotopic and spatiotopic distances between the stimulated sites, we found that the somatotopic distance-more specifically, the distance between and among the sites being defined in cortical topography - determines the strength of thermal referral. Our findings suggest that localization of thermal sensations under thermo-tactile interactions is processed with respect to the somatotopic frame of reference and that this cross-modal processing resides in early cortical areas whose organization conserves topographic information.
\end{abstract}

The thermal sense has been shown to be diffuse and incapable of providing precise spatial information. Thermal sensations are mediated by the small-fiber spinothalamic system, and the neurons on which the spinothalamic fibers terminate have huge receptive fields (Bowsher, 2005; Craig, Chen, Bandy, \& Reiman, 2000; Han, Zhang, \& Craig, 1998; Mountcastle, 1961; Mountcastle \& Powell, 1959; Rose \& Mountcastle, 1959). In addition, the thermal sense summates spatially separate inputs, such that the perceived thermal intensity is a function of the total area of the stimulation, and our capacity to distinguish spatially separate inputs is limited (Cain, 1973; Marks $\&$ Stevens, 1973). As a consequence, when a noncontact thermal stimulus, such as radiant heat, is applied to the skin, the spatial features of the thermal stimulus, such as its location, area, and shape, are poorly resolved (Cain,
1973; Marks \& Stevens, 1973; Stone, 1937; Taus, Stevens, \& Marks, 1975).

It is commonly believed that concurrent tactile inputs facilitate thermal localization. For example, when the hand makes contact with an object, the change in skin temperature and the deformation of the skin activate thermoreceptors and mechanoreceptors located in the skin, and the firing of these receptors transmits the thermal and tactile ${ }^{1}$ inputs to the central nervous system (Kenshalo, 1976). The cross-modal processing of these thermal and tactile inputs facilitates the localization of thermal sensations and is indicated as well by the thermal referral phenomenon, in which the concurrent tactile inputs modify the thermal localization. When observers touched three stimulators simultaneously with the middle three fingers (D2, D3, and D4) but only the

H.-N. Ho, hsinni@avg.brl.ntt.co.jp 
outer two stimulators were cooled or heated, the central (neutral) stimulator was also perceived to be cold or warm (Green, 1977). Interestingly, this illusory thermal sensation disappeared when D3 was withdrawn from the central (neutral) stimulator, clearly indicating that this mislocalization of thermal sensation was induced by the concurrent tactile input received from D3.

In this study, we investigated the cross-modal processing of the thermal and tactile inputs under thermal referral; more specifically, we sought to determine whether the localization under thermo-tactile interaction is processed somatotopically or spatiotopically. Tactile inputs have been shown to be coded somatotopically at early stages of processing and, subsequently, to be processed in spatiotopic space (Azañón \& Soto-Faraco, 2008). The influences from the somatotopic distance have been reported in simple temporal tasks, such as tactile simultaneity judgment (Clark \& Geffen, 1990; Kuroki, Watanabe, Kawakami, Tachi, \& Nishida, 2010) and tactile apparent motion detection (Kuroki et al., 2010; Sherrick, 1968), and in tactile learning (Harris, Harris, \& Diamond, 2001). The influences of spatiotopic distance have been shown in the tasks that require more elaborated processing, such as tactile identification tasks (Haggard, Kitadono, Press, \& Taylor-Clarke, 2006) and tactile spatial attention tasks (Soto-Faraco, Ronald, \& Spence, 2004). The thermal modality has not been studied as thoroughly as the tactile modality, although physiological studies have suggested that thermal sensations are represented somatotopically (Craig, 2002; Craig et al., 2000). As for thermo-tactile interaction, to our knowledge, no study has investigated its frame of reference (somatotopic or spatiotopic). One clue was provided by the study that demonstrated that increasing distance between the stimulated sites reduces the effect of thermal referral (Green, 1978). If the somatotopic distance determines the size of the effect, the localization under thermo-tactile interaction should be represented somatotopically in the early cortical areas whose organization conserves the topographic information. If it is the spatiotopic distance, the localization under thermotactile interaction should be processed in late cortical areas where the spatial coordinate of the environment is defined. Therefore, in the present study, the somatotopic and spatiotopic distances between the stimulated sites were manipulated in order to clarify whether the localization of thermal sensations under thermo-tactile interaction is represented somatotopically or spatiotopically.

\section{METHOD}

\section{Participants}

Ten normal healthy adults ( 5 men and 5 women) between 25 and 39 years of age participated in this experiment. They had no known abnormalities of their tactile and thermal sensory systems, and they were all right-handed. The experiment was conducted in accordance with the ethical standards in the 1964 Declaration of Helsinki. The participants gave their informed consent to participate in the study before the start of the experiment.

\section{Apparatus}

Two thermal displays were constructed to present individually controlled thermal stimuli to different sets of three fingers of two hands.
They were attached to the optical table, and the distance between them could be adjusted as shown in Figure 1. In the present experiment, the thermal displays were either adjacent to one another or separated by a distance of $400 \mathrm{~mm}$. The participants made contact with the stimulators by moving their hands toward the thermal displays from the side. The participants' hands were therefore perpendicular to the optical table during contact with D2 (index finger) at the top and D5 (little finger) at the bottom. The optical table was covered with fabric to cushion and thermally insulate the hands, and the participants' wrists were supported by a sponge pad during contact.

Each thermal display consisted of three Peltier devices with a surface area of $20 \times 20 \mathrm{~mm}$ (FPH1-7106M, Fujitaka Co., Kyoto, Japan) and one dividing spacer with a width of $20 \mathrm{~mm}$. The Peltier devices were housed in plastic holders (labeled P1, P2, and P3). The positions of the holders could be adjusted distally to accommodate different hand shapes and expose a constant surface area of $300 \mathrm{~mm}^{2}$ of the Peltier devices to the participant's fingerpad. In the present experiment, the spacer was placed either next to P3 or between P2 and P3 to manipulate the spatial relationship between the Peltier devices. A platform installed in front of the Peltier devices served as the preparation area for finger positioning before contact.

Two digital-analog converters (ADI16-16 and DA16-16, Contect Co., Osaka, Japan) and a PI control loop programmed in VB.NET were employed to control the surface temperatures of the Peltier devices. The temperature feedback was provided by thermistors (457 $\mu \mathrm{m}$ in diameter and $3.18 \mathrm{~mm}$ in length; 56A1002-C8, Alpha Technics, CA) sandwiched between the Peltier devices and plastic holders. The maximum rate of temperature change was $10^{\circ} \mathrm{C} / \mathrm{sec}$ for cooling and $18^{\circ} \mathrm{C} / \mathrm{sec}$ for heating. Achieving a steady state took about $1 \mathrm{sec}$. After a steady state had been reached, the temperature of each Peltier device could be maintained within $0.5^{\circ} \mathrm{C}$ of the desired temperature. To facilitate heat dissipation, the Peltier devices were placed on top of a copper heat sink (P-200S, Takagi Mfg. Co., Tsukuba, Japan) connected to a water-cooling system (ResaratorXT, Zalman, Seoul, Korea).

To maintain the skin temperature of the hand at $33^{\circ} \mathrm{C}$, a hot plate was constructed by sandwiching a heating wire (YELK-AS, Yagami Inc., Nagoya, Japan) between a $180 \times 180 \mathrm{~mm}$ copper plate and a cupboard. A $0.3-\mathrm{mm}$-thick white sheet of plastic was attached to the top of the copper plate with glue to prevent oxidation of the copper due to constant contact. The surface temperature of the hot plate was controlled with a commercial PID temperature controller (YD-15, Yagami Inc., Nagoya, Japan).

\section{Procedure}

The effects of thermal referral under both cooling and warming were tested with the baseline temperature of the hand maintained at $33^{\circ} \mathrm{C}$. In the cooling session, the participants were presented with one neutral tactile stimulator and a pair of cold stimulators at $25^{\circ} \mathrm{C}$. In the warming session, the participants were presented with one neutral tactile stimulator and a pair of warm stimulators at $39^{\circ} \mathrm{C}$. We chose $25^{\circ} \mathrm{C}$ and $39^{\circ} \mathrm{C}$ as the temperatures of the cooling and warming stimuli because they had been reported to arouse cooling and warming sensations with similar perceived thermal intensity (Greenspan, Roy, Caldwell, \& Farooq, 2003). In addition, the temperature difference between the thermal stimulators and the natural tactile stimulator $\left(8^{\circ} \mathrm{C}\right.$ for cooling and $6^{\circ} \mathrm{C}$ for warming) are well above the thermal discrimination thresholds, which is less than $1^{\circ} \mathrm{C}$ on the fingers and hand (Stevens \& Choo, 1998), so the participants were expected to be able to discriminate the thermal sensations elicited by the stimulators if there was no thermal referral effect.

Three somatotopic distances and two spatiotopic relationships were investigated, giving a total of six experimental conditions, as shown in Figure 2. The somatotopic distances were manipulated by presenting the stimulators to different sets of three fingers of both hands. The levels in the order of increasing somatotopic distance are D2, D3, and D4 of the right hand $\left(\mathrm{S}_{1}\right), \mathrm{D} 2, \mathrm{D} 3$, and D5 of the right hand $\left(\mathrm{S}_{2}\right)$, and D2 and D4 of the right hand and D3 of the left hand $\left(\mathrm{S}_{3}\right)$, in which the stimulated sites in $\mathrm{S}_{1}$ and $\mathrm{S}_{2}$ were ipsilateral, 


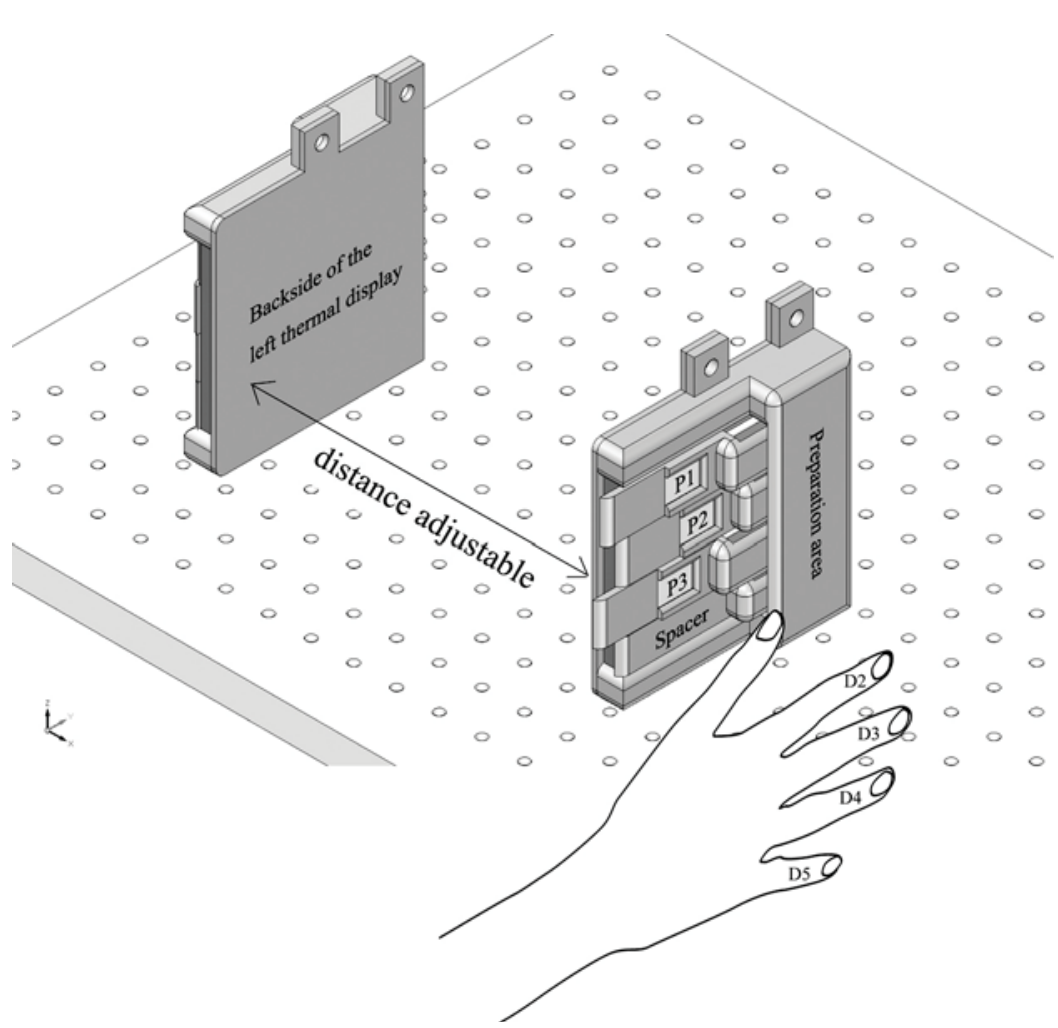

Figure 1. Schematic representation of the experimental setup. Iwo thermal displays were attached to the optical table. The distance between them could be adjusted. The participants made contact with the stimulators by moving their hands toward the thermal displays from the side. Each thermal display consisted of three Peltier devices and one spacer that was used to manipulate the spatial relationship between the Peltier devices. A preparation area was designed to facilitate finger positioning before contact.

whereas those in $\mathrm{S}_{3}$ were bilateral. There were many possible finger combinations for the bilateral condition. The present combination was selected because it ensured that the fingers involved were all somatotopically separated, not only bilaterally (D2 and D4 vs. D3) between the two hands, but also ipsilaterally within the right hand (D2 vs. D4).

The spatiotopic relationship of the stimulated sites was either adjacent or separated. The relationship was manipulated with a spacer in the ipsilateral conditions or with the distance between the thermal displays in the bilateral condition. Under the adjacent condition, the fingers in $\mathrm{S}_{1}$ were at their normal positions. In $\mathrm{S}_{2}$, the participants lifted their D4 and moved their D5 next to the D3 during contact. In $\mathrm{S}_{3}$, the two thermal displays were placed side by side so that the fingers of both hands were adjacent to one another. In the separated condition, the distance between D3 and D4 was increased to $20 \mathrm{~mm}$ in $\mathrm{S}_{1}$. In $\mathrm{S}_{2}$, the fingers were at their normal positions with D3 and D5 spatially separated. In $\mathrm{S}_{3}$, the two thermal displays were $400 \mathrm{~mm}$ apart, so that D3 of the left hand was $400 \mathrm{~mm}$ from D2 and D4 of the right hand.

Each participant came to the lab for 2 days and participated in the cooling session on one day and the warming session on the other. The order of the sessions was counterbalanced among the participants so that half of the participants were tested under cooling and half were tested under warming on the first day. In each session, the six experimental conditions were tested separately in a randomized order. For each experimental condition, the position of the neutral tactile stimulator varied among $\mathrm{P} 1, \mathrm{P} 2$, and $\mathrm{P} 3$, and each neutral stimulator position was repeated four times, to give 12 experimental trials. Six control trials with all the stimulators set to the neutral temperature were also included, giving a block of 18 trials that were presented in randomized order. Each block of trials lasted for about $15 \mathrm{~min}$, and there was at least a 20 -min break between the blocks. With this experimental design, three sets of data were gathered for each experimental condition: the participants' responses with the 12 experimental trials under cooling and warming and the responses with the 12 control trials.

The ambient temperature of the experimental room was maintained at $25^{\circ} \mathrm{C}$. The participants washed their hands with warm water prior to participating in the experiment. Their initial skin temperatures were measured with a thermistor (56A1002-C8, Alpha Technics, CA) and ranged from $30.17^{\circ} \mathrm{C}$ to $33.37^{\circ} \mathrm{C}$.

The participants were informed that two of the three stimulators were at the same temperature and that the temperature of the different one (neutral one) was less cold (warm) than the other two in the cooling (warming) session. The task was to make contact with the three stimulators with the palm side of the first phalanx of their fingers simultaneously. The participants were instructed to bend their fingers to ensure that all the fingerpads were in good contact with the stimulators. A three-alternative forced choice (3AFC) procedure was adopted, in which the participants were instructed to localize the stimulator that felt different from the other two in terms of thermal changes perceived on the fingerpads. The strength of referral was estimated from the participants' performance in localizing the neutral tactile stimulator, with poor performance indicating a strong effect and accurate performance indicating a small effect. To eliminate visual cues, the participants were asked to gaze at a fixation point in front of them, and the thermal displays were arranged in a way that prevented the participants' seeing their 


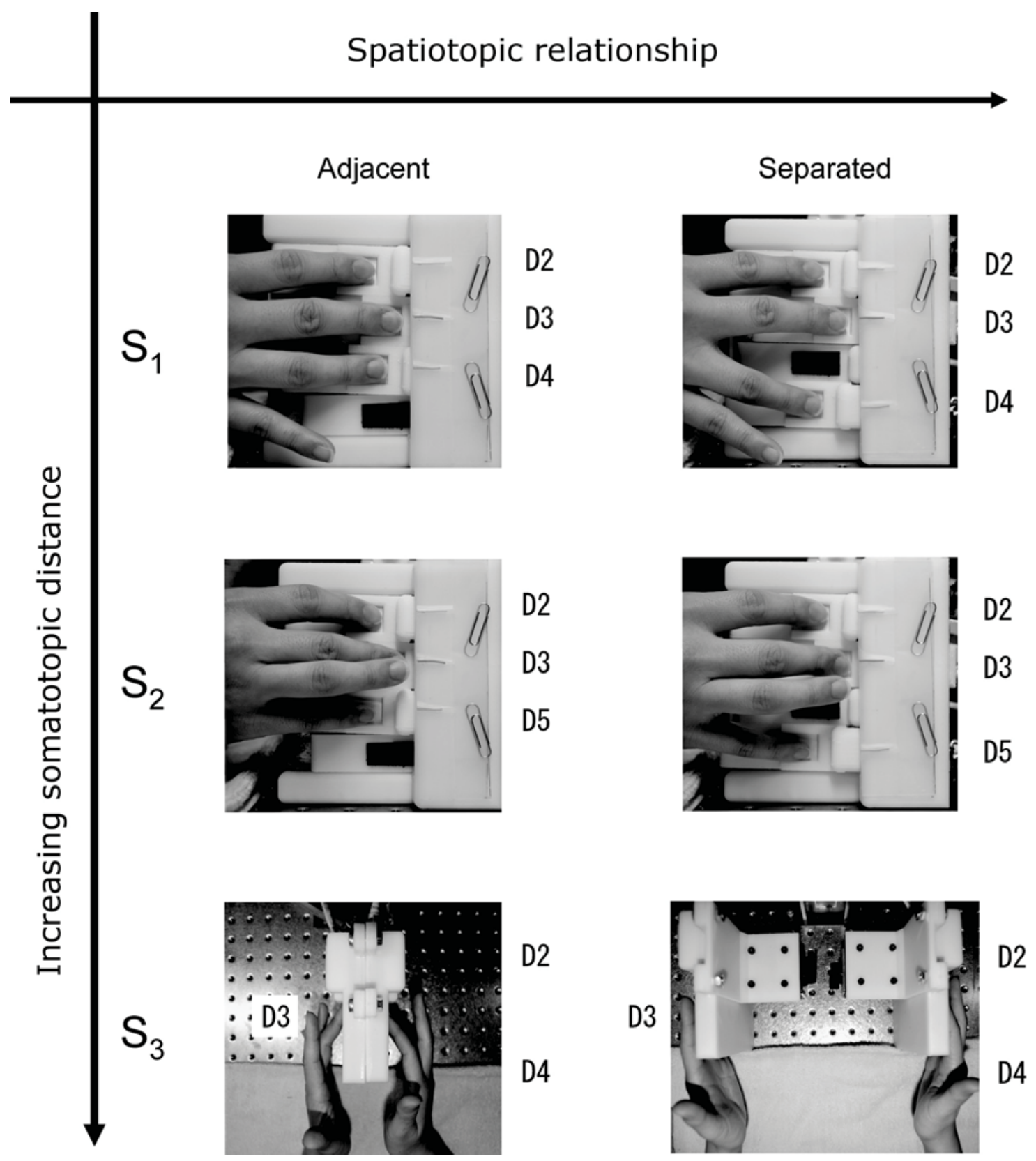

Figure 2. Experimental conditions in the present study. Three somatotopic distances $\left(S_{1}, S_{2}\right.$, and $\left.S_{3}\right)$ and two spatiotopic relationships (adjacent and separated) were investigated, giving a total of six experimental conditions. The participants made contact with the stimulators blindly, so guide wires were used to assist in finger positioning. The task was to report which stimulator felt different from the other two. The photos for conditions $S_{1}$ and $S_{2}$ were taken from the side, and those for condition $S_{3}$ were taken from the top.

own hands and fingers during contact. Given that it was difficult to blindly make contact with the stimulators, guide wires were prepared for each of the experimental conditions, as shown in Figure 2. The participants were instructed to position their fingers by tracing the guide wires before making contact with the stimulators. The experimenter monitored the participants' skin temperatures and their hand movements with an IR camera (SC 640, FLIR) throughout the experiment.

At the beginning of an experimental block, the participants practiced in order to ensure that they were able to blindly make correct contact with the stimulators. Then they placed their hands on the hot plate to adapt their skin temperature to $33^{\circ} \mathrm{C}$. When the participants felt thermal neutrality with the hot plate, they made contact with the stimulators, which had been preset to $33^{\circ} \mathrm{C}$ to establish the temperature corresponding to thermal neutrality. The temperatures of the stimulators were adjusted slightly (on the order of $0.1^{\circ} \mathrm{C}$ ) if $33^{\circ} \mathrm{C}$ evoked a thermal sensation. Once the neutrality had been established, the experiment began. Each trial began with the participants' placing their hands on the hot plate to maintain their skin temperature at $33^{\circ} \mathrm{C}$. Upon hearing a sound cue, the participants positioned their fingers in the preparation area with the help of the guide wires. A second sound cue was then presented, and the par- 
Table 1

Number of Times a Given Position Was Reported As Being Different in the Control Trials

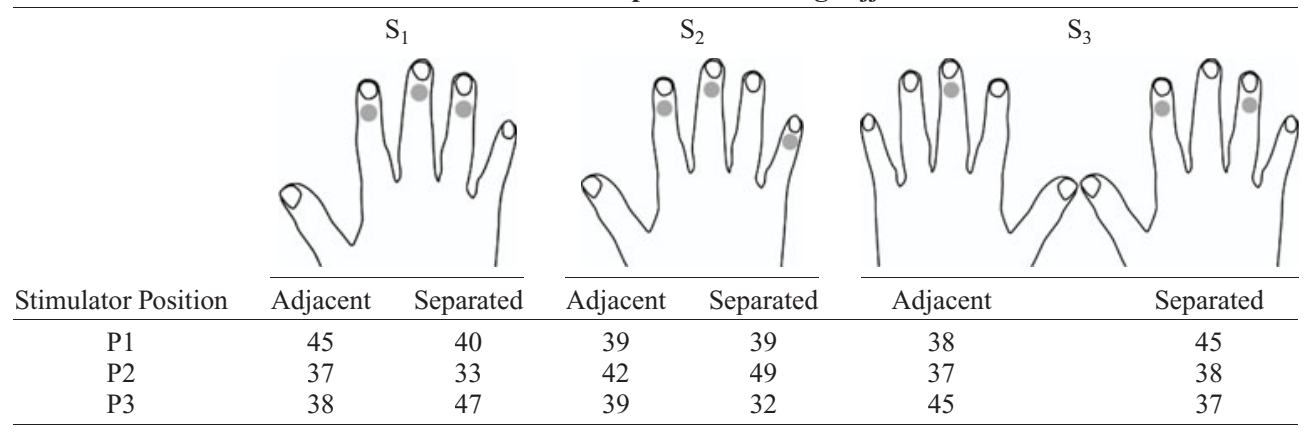

ticipants made contact with the stimulators for $5 \mathrm{sec}$. At the end of the contact, another sound cue was presented, and the participants reported the stimulator that felt different from the other two, using a numerical pad in front of them. The three stimulators were labeled as P1, P2, and P3 from top to bottom, as shown in Figure 1. No feedback was given regarding the correctness of their judgments. The participants then returned their hands to the hot plate to await the start of the next trial. The intertrial interval was around $20 \mathrm{sec}$.

\section{Data Analysis}

The responses to the experimental trials were analyzed in terms of the counts and proportion of correct responses, in which the neutral tactile stimulator-hereafter, termed the target-was correctly localized. Sensory discriminability, $d^{\prime}$, was then estimated from the proportion of correct responses by means of Thurstonian modeling for sensory discrimination, which assumes that two different stimuli generate two sensory distributions with different mean sensations. The standard distance between the two means of the distributions can be used as a measure of sensory discriminability (Bi, 2006; Ennis, 1993). The illusory thermal sensation perceived on the target under thermal referral would introduce confusion to the localization task. The values of $d^{\prime}$ therefore reflect the effect of the thermal referral, with low values indicating a strong effect and high ones indicating a small effect. The $d^{\prime}$ data are approximately normally distributed ( $\mathrm{Bi}$, 2006), which allows parametric statistical analyses. The significant level of the statistical analyses was set at .05. All analyses were performed with SPSS and the MATLAB Statistics Toolbox.

\section{RESULTS}

\section{Position Bias}

Although the $3 \mathrm{AFC}$ procedure can avoid response bias (O’Mahony, 1995; Wickens, 2002), position bias might exist because the participants prefer one of the spatial positions (P1, P2, or P3). To determine whether there was any consistent position bias in the present experiment, we analyzed the number of times a given position was reported as being different in the control trials (all the stimulators set to the neutral temperature). Table 1 shows the pooled data for all the participants. For each experimental condition, the control trials were presented 120 times in total ( 6 repetitions $\times 2$ sessions $\times 10$ participants), and the expected number of times a given position was reported as being different under the assumption of no bias was 40 . A chi-square test was used to compare the data in Table 1 with the expected values, and the results indicated that there was no consistent position bias across the six experimental conditions $(p=.52)$.

\section{Somatotopic Versus Spatiotopic}

Our results showed that somatotopic distance had a significant effect on participants' ability to localize the target. Separate ANOVAs with somatotopic distance and spatiotopic relationship as the within-subjects factors and $d^{\prime}$ as the dependent variable were conducted for cooling and warming, and both revealed main effects of somatotopic distance $[F(2,18)=12.53, p<.001$, for cooling; $F(2,18)=13.19, p<.001$, for warming], indicating that the effect of thermal referral is dictated by the somatotopic distance. The absence of significance for the main effect of the spatiotopic relationship ( $p=.28$ for cooling; $p=$ .75 for warming) and no interaction between effects of the somatotopic distance and spatiotopic relationship ( $p=$ .57 for cooling; $p=.12$ for warming) indicated that the effect of thermal referral was not influenced by whether the stimulated sites were spatiotopically adjacent or separated. As is indicated in Table 2 and Figure 3, participants' discriminability improved with increasing somatotopic distance. Repeated contrast tests revealed that, for cooling, the improvement was not significant when changing from $\mathrm{S}_{1}$ to $\mathrm{S}_{2}(p=.20)$ but reached significance from $\mathrm{S}_{2}$ to $\mathrm{S}_{3}(p<.001)$. For warming, the improvement was close to significance when the somatotopic distance changed from $\mathrm{S}_{1}$ to $\mathrm{S}_{2}(p=.05)$ and reached significance when it changed from $\mathrm{S}_{2}$ to $\mathrm{S}_{3}(p=.03)$. These findings indicate that the bilateral increment in somatotopic distance (from $\mathrm{S}_{2}$ to $\mathrm{S}_{3}$ ) has a more pronounced effect than the ipsilateral increment in the somatotopic distance (from $\mathrm{S}_{1}$ to $\mathrm{S}_{2}$ ).

\section{Cooling Versus Warming}

Besides the influence from the somatotopic distance, Figure 3 also indicates that the sensory discriminability tended to be lower under warming. An ANOVA comparing sensory discriminability under cooling and warming confirmed that there were main effects of thermal stimulation $[F(1,9)=7.52, p=.02]$ and somatotopic distance $[F(2,18)=28.05, p<.001]$, along with a significant three-way interaction among the effects of the thermal stimulation, somatotopic distance, and spatiotopic relationship $[F(2,18)=4.40, p=.03]$. The significant threeway interaction was driven by the significant simple twoway interaction between the effects of the somatotopic distance and thermal stimulation under the separated condition $[F(2,18)=5.17, p=.02]$. To understand the 
Table 2

Group Mean Proportion of Correct Responses, $p(c)$, and the Corresponding Sensory Discriminability, $d^{\prime}$, in Localizing the Target, Regardless of Target Position

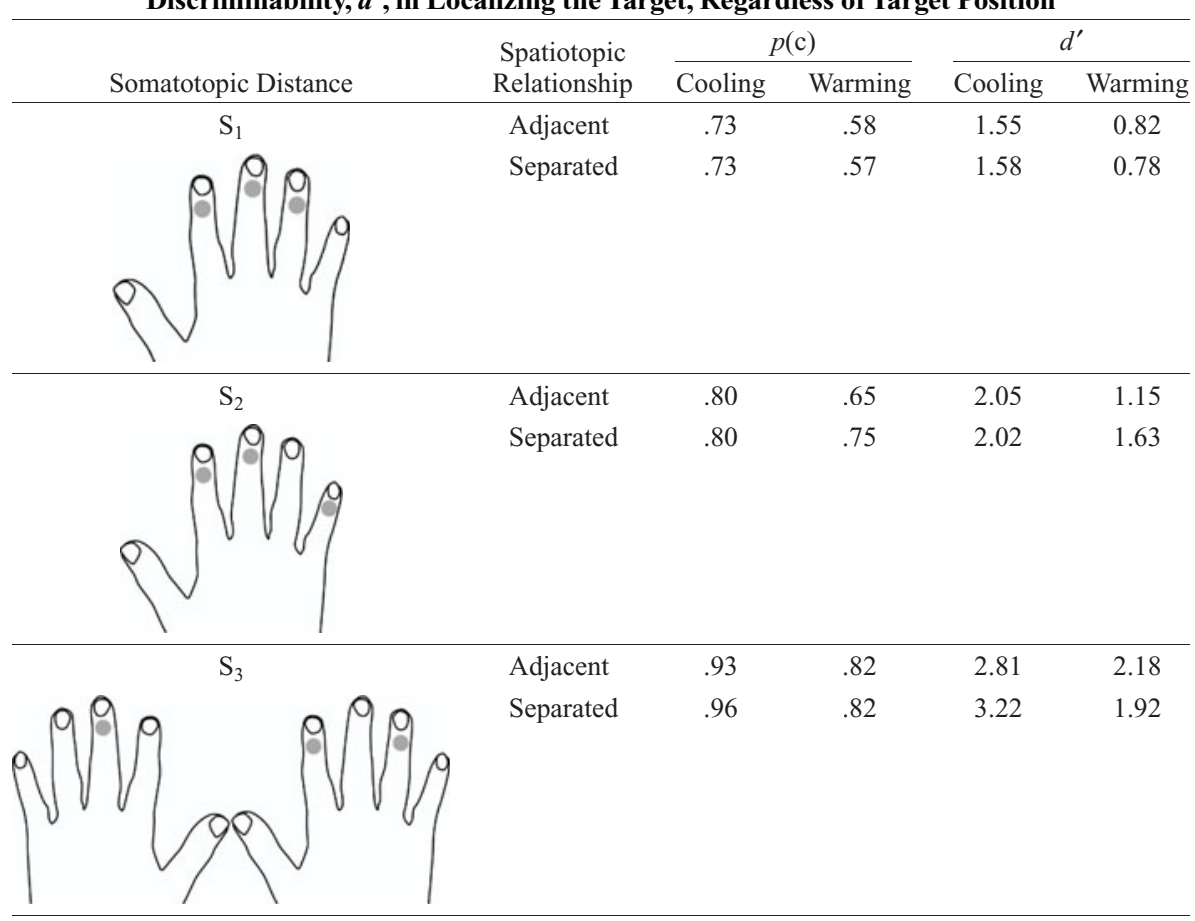

nature of this interaction, we tested for simple main effects between cooling and warming under each somatotopic distance in the separated condition. The results indicated that the discriminability was significantly worse under warming than under cooling in $\mathrm{S}_{1}[F(1,18)=16.58, p<$ $.001]$ and $\mathrm{S}_{3}[F(1,18)=42.64, p<.001]$. These findings suggest that the effect of thermal referral is stronger under warming.

\section{Confusion Matrices}

Given that there was no consistent position bias, as was shown in the previous analysis, and that the thermal sensitivities among the fingers were similar (Schoen, Marks, \& Green, 2004), we analyzed the confusion matrices of the participants' responses to find clues as to whether the thermal referral effect depended on which finger was making contact with the neutral tactile stimu-
A

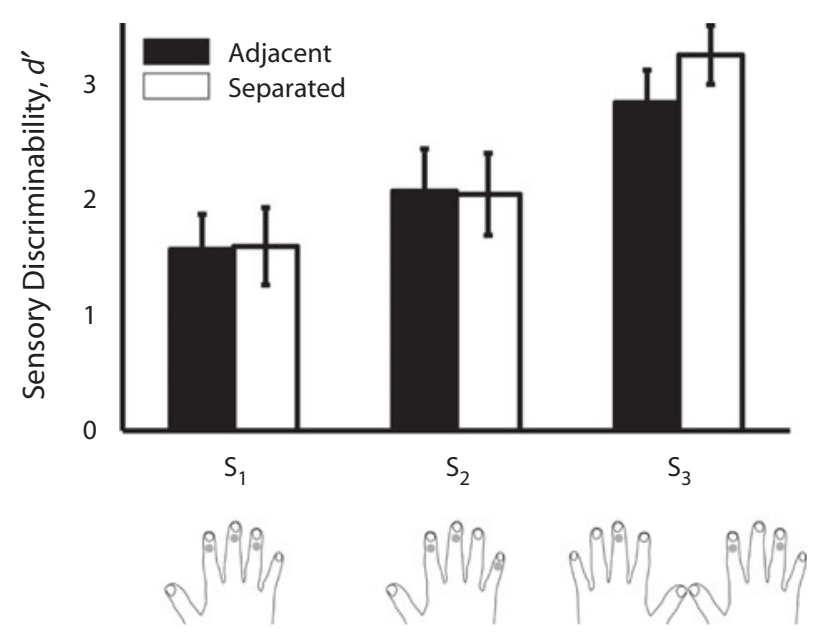

B
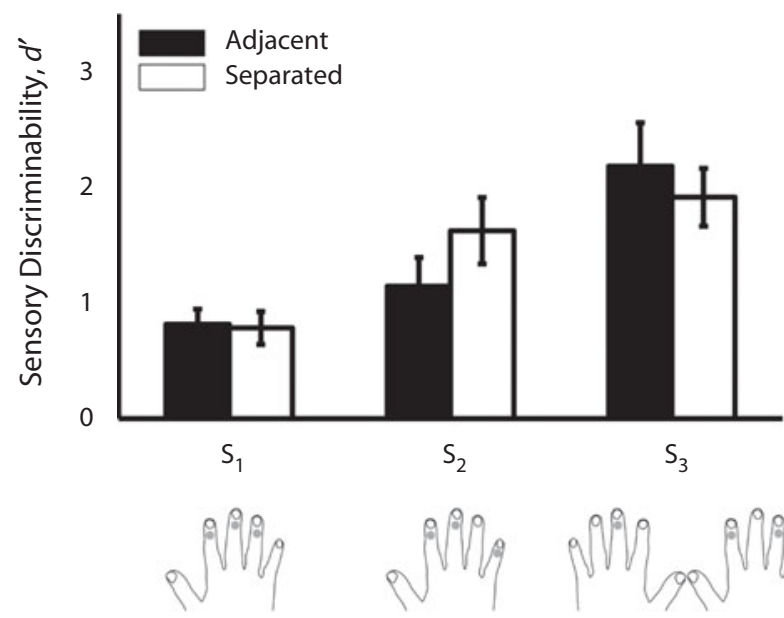

Warming

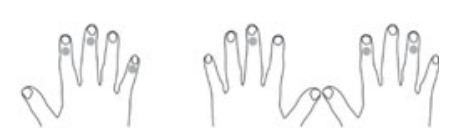

Figure 3. Group mean discriminability $\left(d^{\prime}\right)$ in localizing the target as a function of the somatotopic distance and spatiotopic relationship under cooling (A) and warming (B). Trials in which the stimulators were spatiotopically adjacent are shown in black; those in which they were separated are shown in white. Error bars show the standard errors of the means. 
lator - hereafter, termed the target finger. Given that the spatiotopic relationship did not have a significant effect, the participants' responses were pooled for the adjacent and separated conditions under each somatotopic distance, and the scores in the confusion matrices were expressed as the proportions of times a given finger was reported as the target finger. As is shown in Figure 4, we used radar plots to visualize the confusion matrices. Each spoke in the plots represents the proportion of times the corresponding finger was reported as the target finger, and the shaded area represents the participants' responses with a given target finger. If the participants performed accurately, the shaded area would be shaped like a spike with its tip located at the spoke of the target finger. On the other hand, if there was confusion in localization, the shaded area would be shaped like a triangle.

Figure 4 indicates that the participants' performance depended on the target finger and that they performed worst when the target finger was D3. Chi-square tests were conducted to examine target finger dependence by comparing the observed confusion matrix with the one expected under the assumption of no dependence. ${ }^{2}$ The results revealed that the dependence was significant in $\mathrm{S}_{1}$ and $\mathrm{S}_{2}$ under both cooling and warming $(p<.001$ for all conditions). These results indicated that the thermal referral effect was strongest when the thermal sensations were referred toward D3 in the ipsilateral conditions $\left(\mathrm{S}_{1}\right.$ and $\mathrm{S}_{2}$ ). In addition, a difference in how the participants mislocalized the target was found between cooling and warming. Under warming, the participants' responses were biased toward D2, whereas under cooling, such bias did not exist.

Chi-square tests also indicated that there was no target finger dependence in the bilateral condition $\left(\mathrm{S}_{3}\right)$ under both cooling and warming. In addition, the performance with D3 of the contralateral hand was almost perfect $(96 \%$ correct for cooling; $90 \%$ correct for warming), indicating that the thermal sensations did not occur across hands. The performance with D2 and D4 of the right hand as the target fingers was quite different under cooling and warming, with $91 \%$ and $95 \%$ correct under cooling but only $83 \%$ and $73 \%$ correct under warming. These findings revealed that thermal referral had virtually no effect between D2 and D4 under cooling but a small effect under warming, demonstrating that thermal referral under cooling was more limited by the somatotopic distance.

\section{DISCUSSION}

\section{Principal Findings}

Under the present experimental conditions, the strength of the mislocalization under thermal referral was a function of somatotopic distance-more specifically, the distance between and among the sites represented in the cortical topography. Whereas the ipsilateral increment of the somatotopic distance (within one hand) reduced the effect, the increment of the somatotopic distance across the midline (across hands) diminished the effect completely. These findings indicate that the cross-modal processing between thermal and tactile modalities for localization resides in early cortical areas whose organization conserves the topographic information.

Whether the stimulated sites are spatiotopically adjacent or separated was shown to exert no influence on the strength of thermal referral, indicating that thermal sensations are represented somatotopically, rather than spatiotopically. Although we typically associate the thermal sensations elicited upon making contact with an object and use this information for object recognition, thermal sensations are ultimately feelings from the body that signal the physiological conditions (Craig, 2002). This functional significance is reflected in the somatotopic, rather than spatiotopic, representation of the thermal sensations.

\section{Difference Between Cooling and Warming Under Thermal Referral}

The difference in spatial acuity presumably contributes to the difference between cooling and warming under thermal referral. Our sense of warmth has been shown to be more diffuse than our sense of cold in a spatial discrimination task (Hensel, 1982; Lee, McGillis, \& Greenspan, 1996), and the spatial summation is greater for warming stimuli than for cooling stimuli (Greenspan \& Kenshalo, 1985). It is also known that cold receptors are always more numerous than warm receptors in different body regions (Darian-Smith, 1984) and that cooling the skin activates certain mechanoreceptors that are sensitive to touch (Hensel, 1981; Spray, 1986). All of these factors can contribute to the relative good spatial acuity of the cold and the limited thermal referral under cooling.

\section{Finger Selectivity Under Thermal Referral}

Thermal sensations were referred more strongly toward D3 in the ipsilateral conditions. Replacing the ipsilateral D3 with the contralateral D3 in $S_{3}$ completely removed the effect, indicating that the finger selectivity is associated with the somatotopic representation of the fingers. The preference for D3 reflected that the strength of thermal referral is determined by the degree of overlap between and among the finger representations in the cortical areas. When the neutral site is located at the center, it is overlapped by the cooled/heated sites on either side. However, when the neutral site is moved to the side, the degree of overlapping decreases, and, as a result, the effect of thermal referral is reduced. The tendency to mislocalize D3 to D2, rather than to D4 or D5, under warming could be explained by the fact that D3 and D2 are innervated by the same nerve (median nerve), whereas D4 is innervated by both the median and ulnar nerve and D5 is innervated by the ulnar nerve alone. It has been shown that both cooling and warming stimuli are more precisely localized when they are separated transdermatomally than when they are separated intradermatomally (Lee et al., 1996). However, the same tendency was not found under cooling in the present study.

\section{Possible Cortical Representation}

It still remains unclear which cortical areas are involved in this cross-modal processing. It has been shown 


\section{Cooling}

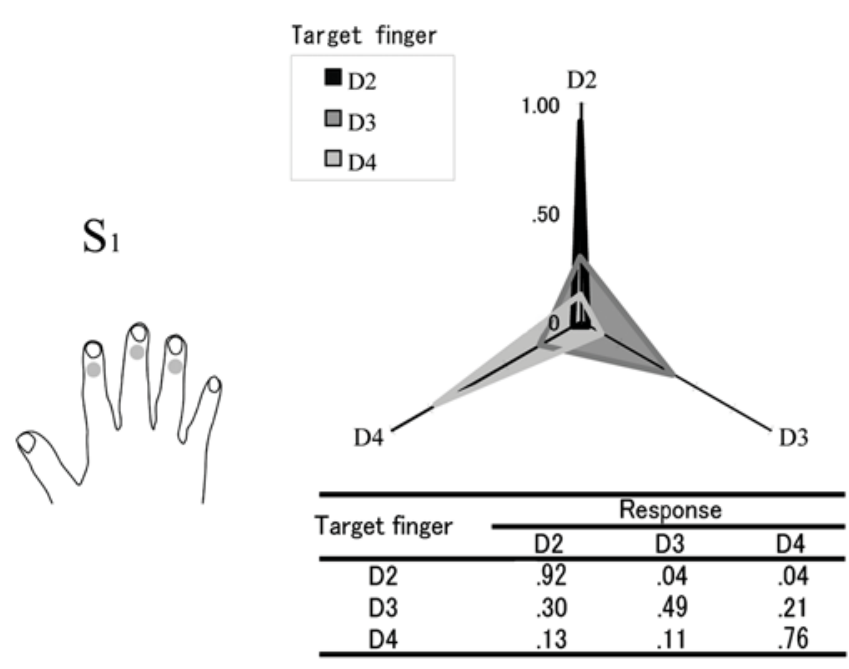

$\mathrm{S}_{2}$

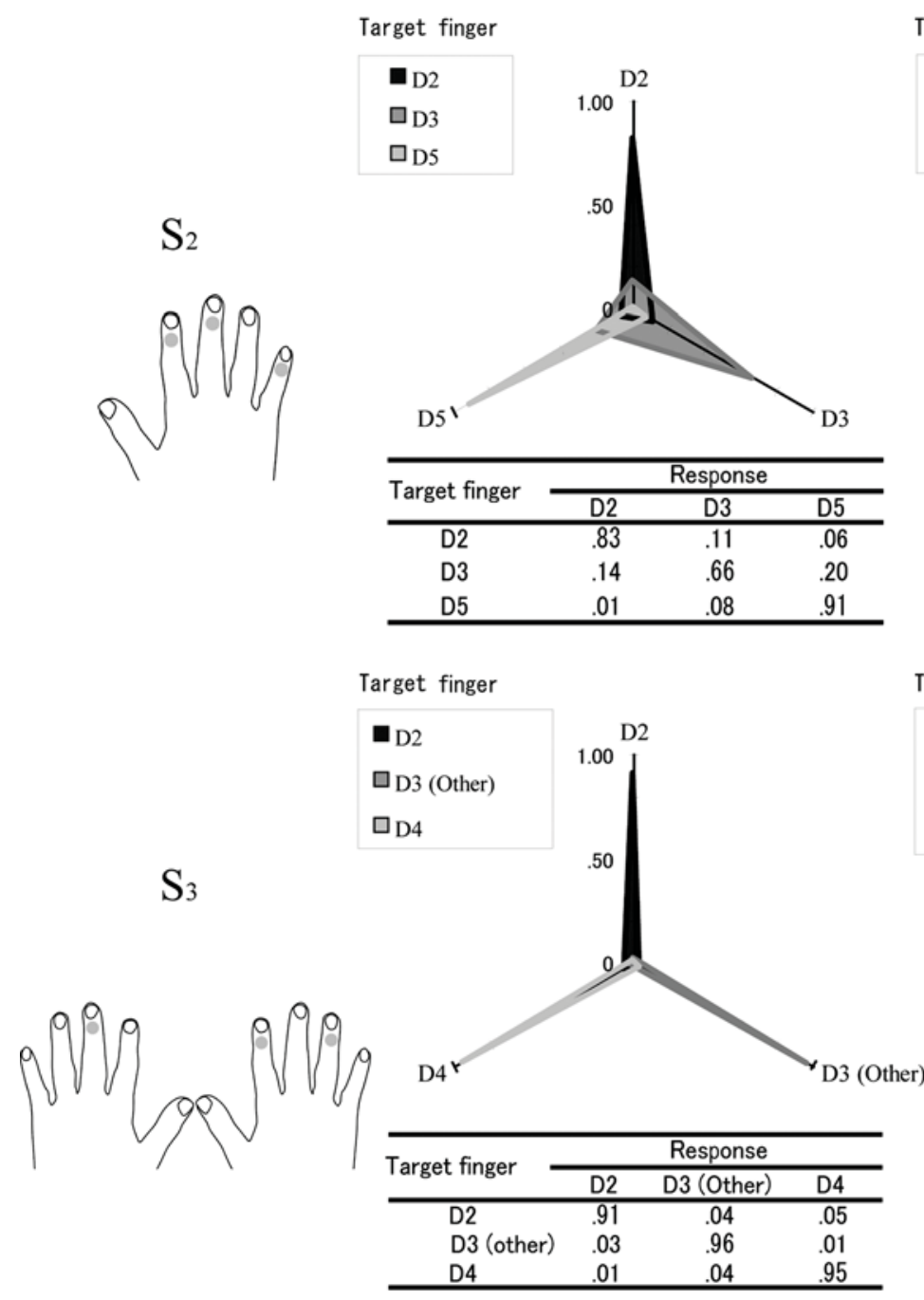

Target finger

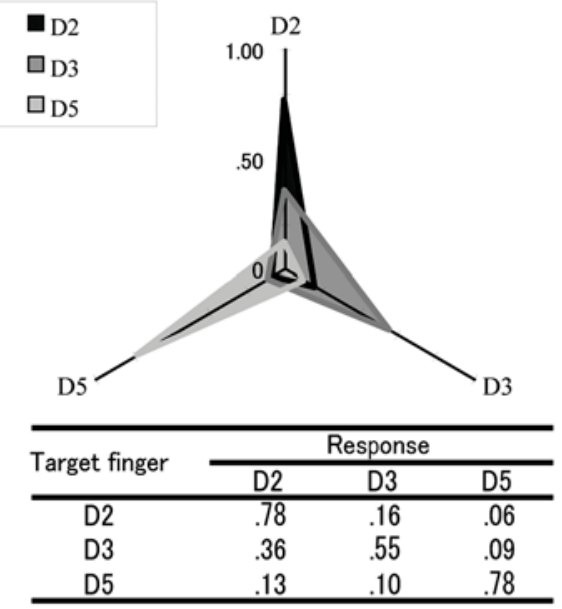

Target finger

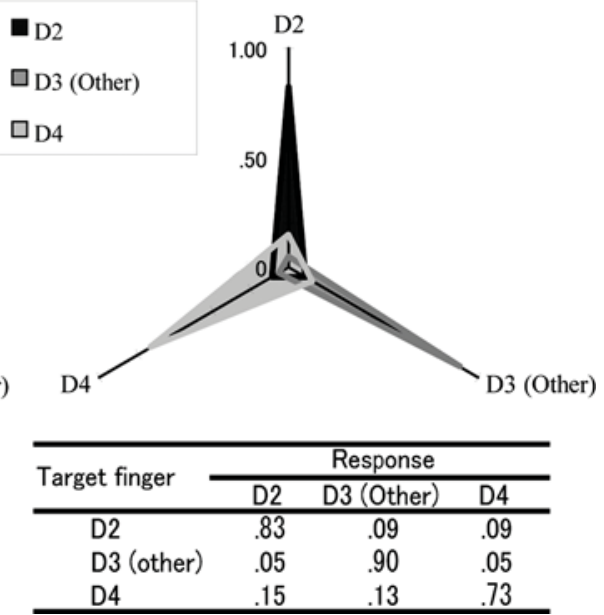

Figure 4. Confusion matrices of each somatotopic distance under cooling and warming, with scores expressed in proportions and their corresponding radar plots. "Other" indicates the finger of the opposite hand. 
that thermal and tactile sensory systems have physiologically separate ascending sensory pathways (Darian-Smith, 1984) and that their representations in the brain occupy different cortical areas: Discriminative tactile sensations are mediated by the somatosensory cortex, whereas the haptic capacity of thermal sensations is subserved by the dorsal posterior insular cortex (Craig et al., 2000; Hua, Strigo, Baxter, Johnson, \& Craig, 2005). The simultaneous tactile and thermal inputs received from touch are therefore represented in two distinct somatotopic maps, indicating that this cross-modal processing occurs somewhere beyond the primary thermal and tactile sensory cortices. It is speculated that the median insula is involved, given that the thermoreceptive neurons at lamina I project topographically to the mid/posterior dorsal insula by way of the ventromedial nucleus, VMpo (Craig, Bushnell, Zhang, \& Blomqvist, 1994; Craig et al., 2000), and that the median insula has been shown to respond to integrative inputs, such as those from the tactile and premotor regions (Augustine, 1996; Olausson et al., 2002). However, further neurophysiological investigations are needed to identify the exact cortical areas involved.

\section{Role of Touch in Thermal Perception}

The phenomenon of thermal referral investigated in the present study demonstrated the influence of touch on thermal localization. The study conducted by Green (2009) also showed that active touch can attenuate the innocuous and nociceptive thermal sensations on the hand. These phenomena demonstrate that haptic thermal perception is not determined solely by the properties of the thermal stimulus. Touch also plays a fundamental role in how we perceive temperature. It is thus important to understand the cross-modal processing between thermal and tactile modalities in order to deepen our knowledge about information processing during manual exploration, which is essential for acquiring information from the external world.

\section{AUTHOR NOTE}

Coauthor H. A. is currently affiliated with Osaka University, Suita, Osaka, Japan. Correspondence concerning this article should be addressed to H.-N. Ho, Human and Information Science Laboratory, NTT Communication Science Laboratories, NTT Corporation, 3-1 Morinosato Wakamiya, Atsugi, Kanagawa, 243-0198, Japan (e-mail: hsinni@avg.brl.ntt.co.jp).

\section{REFERENCES}

Augustine, J. R. (1996). Circuitry and functional aspects of the insular lobe in primates including humans. Brain Research Reviews, 22, 229-244.

Azañón, E., \& Soto-Faraco, S. (2008). Changing reference frames during the encoding of tactile events. Current Biology, 18, 1044-1049. doi:10.1016/j.cub.2008.06.045

BI, J. (2006). Sensory discrimination tests and measurements. Ames, IA: Blackwell.

Bowsher, D. (2005). Representation of somatosensory modalities in pathways ascending from the spinal anterolateral funiculus to the thalamus demonstrated by lesions in man. European Neurology, 54, 14-22. doi: $10.1159 / 000086884$

CAIN, W. S. (1973). Spatial discrimination of cutaneous warmth. American Journal of Psychology, 86, 169-181.

Clark, C. R., \& GefFen, G. M. (1990). Hemispheric equivalence for simultaneity judgments of somatosensory stimuli. Cognitive Neuropsychology, 7, 311-327.

Craig, A. D. (2002). How do you feel? Interoception: The sense of the physiological condition of the body. Nature Reviews Neuroscience, $\mathbf{3}$, 655-666. doi:10.1038/nrn894

Craig, A. D., Bushnell, M. C., Zhang, E.-T., \& Blomevist, A. (1994). A thalamic nucleus specific for pain and temperature sensation. Nature, 372, 770-773.

Craig, A. D., Chen, K., Bandy, D., \& Reiman, E. M. (2000). Thermosensory activation of insular cortex. Nature Neuroscience, 3, 184-190. doi: $10.1038 / 72131$

DARIAN-SMith, I. (1984). Thermal sensibility. In I. Darian-Smith (Ed.), Handbook of physiology: The nervous system (pp. 879-913). Bethesda, MD: American Physiological Society.

ENNIS, D. M. (1993). The power of sensory discrimination methods. Journal of Sensory Studies, 8, 353-370.

Green, B. G. (1977). Localization of thermal sensation: An illusion and synthetic heat. Perception \& Psychophysics, 22, 331-337.

GREEN, B. G. (1978). Referred thermal sensations: Warmth versus cold. Sensory Processes, 2, 220-230.

Green, B. G. (2009). Temperature perception on the hand during static versus dynamic contact with a surface. Attention, Perception, \& Psychophysics, 71, 1185-1196. doi:10.3758/APP.71.5.1185

Greenspan, J. D., \& Kenshalo, D. R. (1985). The primate as a model for the human temperature-sensing system: 2 . Area of skin receiving thermal stimulation. Somatosensory Research, 2, 315-324.

Greenspan, J. D., Roy, E. A., Caldwell, P. A., \& Faroog, N. S. (2003). Thermosensory intensity and affect throughout the perceptible range. Somatosensory \& Motor Research, 20, 19-26. doi:10 $.1080 / 0899022031000083807$

Haggard, P., Kitadono, K., Press, C., \& Taylor-Clarke, M. (2006). The brain's fingers and hands. Experimental Brain Research, 172, 94102. doi: $10.1007 / \mathrm{s} 00221-005-0311-8$

Han, Z. S., Zhang, E. T., \& CraIG, A. D. (1998). Nociceptive and thermoreceptive lamina I neurons are anatomically distinct. Nature Neuroscience, 1, 218-225.

Harris, J. A., Harris, I. M., \& Diamond, M. E. (2001). The topography of tactile learning in humans. Journal of Neuroscience, 21, 10561061.

HENSEL, H. (1981). Thermoreception and temperature regulation. New York: Academic Press.

Hensel, H. (1982). Thermal sensations and thermoreceptors in man. Springfield, IL: Thomas.

Hua, L. H., Strigo, I. A., Baxter, L. C., Johnson, S. C., \& Craig, A. D. (2005). Anteroposterior somatotopy of innocuous cooling activation focus in human dorsal posterior insular cortex. American Journal of Physiology, 289, 319-325. doi:10.1152/ajpregu.00123.2005

Kenshalo, D. R. (1976). Correlations of temperature sensitivity in man and monkey, a first approximation. In Y. Zotterman (Ed.), Sensory functions of the skin with special reference to man (pp. 305-330). Oxford: Pergamon.

Kuroki, S., Watanabe, J., Kawakami, N., Tachi, S., \& Nishida, S. (2010). Somatotopic dominance in tactile temporal processing. Experimental Brain Research, 203, 51-62.

Lee, D. K., McGillis, S. L. B., \& Greenspan, J. D. (1996). Somatotopic localization of thermal stimuli: I. A comparison of withinversus across-dermatomal separation of innocuous thermal stimuli. Somatosensory \& Motor Research, 13, 67-71.

Marks, L. E., \& Stevens, J. C. (1973). Spatial summation of warmth: Influence of duration and configuration of the stimulus. American Journal of Psychology, 86, 251-267.

Mountcastle, V. B. (1961). Duality of function in the somatic afferent system. In M. A. B. Brazier (Ed.), Brain and behavior (pp. 67-93). Washington, DC: American Institute of Biological Science.

Mountcastle, V. B., \& Powell, T. P. S. (1959). Neural mechanisms subserving cutaneous sensibility. Bulletin of the Johns Hopkins Hospital, 105, 201-232.

Olausson, H., Lamarre, Y., Backlund, H., Morin, C., Wallin, B. G., StarcK, G., ET AL. (2002). Unmyelinated tactile afferents signal touch and project to insular cortex. Nature Neuroscience, 5, 900-904. doi: $910.1038 / \mathrm{nn} 1896$

O'Mahony, M. (1995). Who told you the triangle test was simple? Food Quality \& Preference, 5, 227-238. 
Rose, J. E., \& Mountcastle, V. B. (1959). Touch and kinesthesis. In J. Field (Ed.), Handbook of physiology: Section 1. Neurophysiology (Vol. 1, pp. 387-429). Washington, DC: American Physiological Society.

Schoen, K. L., Marks, L. E., \& Green, B. G. (2004). Temperature perception throughout the hand. Paper presented at the Society for Neuroscience Abstracts, Program No. 59.14, San Diego, CA.

SherricK, C. E. (1968). Bilateral apparent haptic movement. Perception \& Psychophysics, 4, 159-160.

Soto-Faraco, S., Ronald, A., \& Spence, C. (2004). Tactile selective attention and body posture: Assessing the multisensory contributions of vision and proprioception. Perception \& Psychophysics, 66, 1077-1094.

SpRAY, D. C. (1986). Cutaneous temperature receptors. Annual Review of Physiology, 48, 625-638.

Stevens, J. C., \& Choo, K. C. (1998). Temperature sensitivity of the body surface over the life span. Somatosensory \& Motor Research, 15, 13-28.

Stone, L. J. (1937). An experimental study of form perception in the thermal senses. Psychophysical Record, 1, 234-337.

Taus, R. H., Stevens, J. C., \& Marks, L. E. (1975). Spatial localization of warmth. Perception \& Psychophysics, 17, 194-196.

Wickens, T. D. (2002). Elementary signal detection. New York: Oxford University Press.

\section{NOTES}

1. The term tactile in the present study refers to the processes and sensations associated with stimulation of the mechanoreceptors.

2. Please see the Appendix for the derivation of the expected confusion matrix.

\section{Derivation of the Expected Confusion Matrix Under the "No Dependence" Assumption}

As an example, we show in Table A1 the confusion matrix for $\mathrm{S}_{1}$ under cooling, with the scores expressed in counts. (The bold values in the tables below indicate the correct responses.)

Table A1

Confusion Matrix for $S_{1}$

Under Cooling

\begin{tabular}{crrr}
\hline Target & \multicolumn{3}{c}{ Response } \\
\cline { 2 - 4 } Finger & D2 & D3 & D4 \\
\hline D2 & $\mathbf{7 4}$ & 3 & 3 \\
D3 & 24 & $\mathbf{3 9}$ & 17 \\
D4 & 10 & 9 & $\mathbf{6 1}$ \\
\hline
\end{tabular}

The pooled proportion of correct responses is first calculated by dividing the total number of correct responses by the number of trials presented in the given condition. In this example, it is $(74+39+61) / 240=0.725$. With 80 trials for each target finger, the expected proportion of correct responses is $0.725 \times 80=58$, and the expected confusion matrix is defined in Table A2.

Table A2

Expected Confusion Matrix

for $\mathrm{S}_{1}$ Under Cooling

\begin{tabular}{cccc}
\hline Target & \multicolumn{3}{c}{ Response } \\
\cline { 2 - 4 } Finger & D2 & D3 & D4 \\
\hline D2 & $\mathbf{5 8}$ & 11 & 11 \\
D3 & 11 & $\mathbf{5 8}$ & 11 \\
D4 & 11 & 11 & $\mathbf{5 8}$ \\
\hline
\end{tabular}

(Manuscript received January 16, 2009; revision accepted for publication March 25, 2010.) 